\title{
Análise de Frequência dos Dados Pluviométricos Observados em 2011 e 2013 na Região Serrana, Estado do Rio de Janeiro
}

\author{
Clarisse R. Ottero $^{1}$ iD, Leonardo Tristão Chargel $^{2}[\mathrm{D}$, \\ Mônica de Aquino Galeano Massera da $\operatorname{Hora}^{3}$ iD \\ ${ }^{1}$ Departamento de Engenharia Agrícola e do Meio Ambiente, \\ Universidade Federal Fluminense, Niterói, RJ, Brasil. \\ ${ }^{2}$ Instituto Estadual do Ambiente, Rio de Janeiro, RJ, Brasil. \\ ${ }^{3}$ Departamento de Engenharia Agrícola e do Meio Ambiente, \\ Universidade Federal Fluminense, Niterói, RJ, Brasil.
}

Recebido em 2 de Maio de 2017 - Aceito em 11 de Outubro de 2017

Equação no texto

\begin{abstract}
Resumo
As chuvas ocorridas em janeiro de 2011 na Região Serrana provocaram o que foi considerado o pior desastre da história do país, com mais de 900 mortos. Já as chuvas de março de 2013, com total pluviométrico superior, acarretaram 33 óbitos. O presente trabalho buscou comparar os dois eventos, a partir de dados horários do Sistema de Alerta do Instituto Estadual do Ambiente e de registros históricos diários da Agência Nacional de Águas. Os dados diários foram convertidos por fator de desagregação específico e ajustou-se o modelo de distribuição de probabilidades de Gumbel. Em Nova Friburgo, o tempo de recorrência do evento de 2011 foi de 150 anos. Já para Petrópolis e Teresópolis, o evento de 2013 resultou em tempos de retorno iguais a 135 e 115 anos, respectivamente. Apesar das recorrências de mesma ordem de grandeza entre os desastres associados, os eventos resultaram em efeitos distintos nas cidades estudadas. Recomenda-se que na avaliação da criticidade de um desastre não se considere apenas o total precipitado, visto que fatores como relevo, umidade do solo e ocupação de áreas de risco, bem como ocorrência de deslizamentos anteriores, podem ser determinantes na análise.
\end{abstract}

Palavras-chave: chuvas intensas, desastres, Petrópolis, Nova Friburgo.

\section{Frequency Analysis of Rainfall Data Observed in 2011 and 2013 in Serrana Region, Rio de Janeiro State}

\begin{abstract}
The rain that fell in the Serrana region in January 2011 caused what was considered to be the worst disaster in the history of Brazil, with more than 900 deaths. On the other hand, the rain that fell in March 2013, which was a larger total, gave rise to 33 deaths. The present study sought to compare these two events, using hour-by-hour data from the warning system of the State Environmental Institute and the historical daily records of the National Water Agency. The daily data were converted using a specific disaggregation factor and were fitted to Gumbel's probability distribution model. In Nova Friburgo, the recurrence interval for the 2011 event was 150 years. For Petrópolis and Teresópolis, the recurrence intervals for the 2013 event were 135 and 115 years, respectively. Despite recurrence intervals of the same order of magnitude, the disasters that were associated with these events had different effects in the municipalities studied. It is recommended that assessments on the criticality of a disaster should not be based solely on the precipitation, given that factors such as relief, soil moisture content, land occupation in areas at risk and previous occurrences of landslides may be determinants in the analysis.
\end{abstract}

Keywords: extreme rainfall, disasters, Petropolis, Nova Friburgo.

Autor de correspondência: Mônica de Aquino Galeano Massera da Hora, dahora@vm.uff.br. 


\section{Introdução}

De acordo com os dados do International Disaster Database (EM-DAT), entre 1990 e 2014, a ocorrência de inundações representou a maior parte dos desastres brasileiros reportados internacionalmente. Em segundo lugar, estão os desastres relacionados aos deslizamentos (CRED, 2016).

Das Unidades da Federação, o Estado do Rio de Janeiro é o que mais se destaca, principalmente, no evento relacionado ao megadesastre da Região Serrana, ocorrido no ano de 2011, quando o número de vítimas fatais impactou o total de óbitos por desastres no Brasil (Carmo e Anazawa, 2014).

A região da Serra do Mar, com seus compartimentos montanhosos e alinhamentos serranos, torna-se particularmente vulnerável à entrada de massas de ar e às tempestades associadas. Consequentemente, eventos extremos de chuvas intensas seguidas de movimentos de massa e inundações são recorrentes (Waldherr e Tupinambá, 2014). Antes do desastre de 2011, o Conselho Regional de Engenharia do Rio de Janeiro (CREA-RJ) havia realizado dois levantamentos na região, apontando que, aproximadamente, 42 mil moradores viviam em 230 áreas vulneráveis, onde foram construídas 10 mil casas (Busch e Amorim, 2011).

Entre os dias 11 e 12 de janeiro de 2011, chuvas de intensidade significativa, provocadas pela entrada de massas de ar provenientes da Zona de Convergência do Atlântico Sul na Região Serrana, deflagraram o que seria considerado o pior desastre natural ocorrido em território brasileiro, até então, causando 905 mortes e afetando mais de 300 mil pessoas, $42 \%$ da população dos municípios atingidos (Banco Mundial, 2012). Na época do desastre, a mídia divulgou que em Teresópolis o temporal foi o pior evento de chuvas desde janeiro de 1961, quando a chuva de $24 \mathrm{~h}$ totalizou 161,6 $\mathrm{mm}$. Também foi divulgado que a maior chuva anterior ao desastre em Nova Friburgo ocorreu em 1964, quando o índice pluviométrico máximo foi de $113 \mathrm{~mm}$ em $24 \mathrm{~h}$. Em Petrópolis, o evento superou o volume de chuvas de $24 \mathrm{~h}(154,4 \mathrm{~mm})$ observado em 25 de janeiro de 1947 (Lauriano et al., 2011).

No entanto, a magnitude dos desastres de origem pluviométrica não é consequência apenas do total precipitado. No Brasil, os desastres naturais tendem a estar relacionados a fenômenos climáticos potencializados pela ação do homem, estabelecendo uma relação entre o avanço da degradação ambiental, a intensidade do impacto dos desastres e o aumento da vulnerabilidade humana (Maffra e Mazola, 2007).

Como exemplo, menciona-se o evento ocorrido entre os dias 17 e 18 de março de 2013, que foi marcado por chuvas intensas e prolongadas influenciadas pelas nuvens carregadas da Zona de Convergência de Umidade, que canalizou muita umidade entre o Centro-Oeste e o Sudeste do Brasil, e sua somatória à ação dos ventos úmidos vindos do oceano (DRM-RJ, 2013). De acordo com informações da Secretaria de Estado da Defesa Civil (SEDEC), fortes chuvas atingiram os municípios de Angra dos Reis, Mangaratiba, Niterói, Teresópolis e Petrópolis, sendo este último o mais afetado. Em Petrópolis, no posto Quitandinha instalado no bairro de mesmo nome, a precipitação acumulada em $24 \mathrm{~h}$ foi igual a 407,2 mm (Rio de Janeiro, 2013a). Em decorrência do evento, na cidade foram observados escorregamentos generalizados e registrados 33 óbitos (Rio de Janeiro, 2013b).

A fixação de um valor para se estabelecer chuvas intensas é difícil, uma vez que o impacto pode ser diferente, dependendo do local de incidência, seja em áreas rurais ou urbanas (Pinto, 1999). Vários autores, entre eles Huff e Neill (1959), Damé et al. (1996), Silva et al. (2002) e Thebaldi (2012), buscaram ajustar dados de chuva observados a modelos teóricos de distribuição de probabilidade. De acordo com Hartmann et al. (2011), a literatura tem mostrado que a distribuição de probabilidade de Gumbel é a que melhor se ajusta à precipitação pluvial máxima, tal como pode ser observado nos trabalhos de Pfafstetter (1957), Hershfield (1961) e Eltz et al. (1992) e constatado em Silva et al. (2002) e Thebaldi (2012) que concluíram que o modelo de Gumbel foi o que melhor representou os dados de precipitação diária máxima anual.

O presente estudo apresenta uma análise do tempo de recorrência das chuvas ocorridas na Região Serrana, comparando a magnitude dos eventos de janeiro de 2011 e março de 2013, através do levantamento das séries de precipitação máxima das estações pluviométricas disponíveis e da avaliação da sua frequência de excedência pela distribuição de probabilidade de Gumbel.

\section{Materiais e Métodos}

Os dados de precipitação utilizados neste trabalho referem-se aos observados em nível diário na rede pluviométrica da Agência Nacional de Águas (ANA) e aos observados em nível horário no Sistema de Alerta de Cheias do Instituto Estadual do Ambiente (Inea).

Para o estudo da frequência de ocorrência de precipitações intensas, para os tempos de retorno de 5, 10, 20, 50, 100 e 1000 anos, ajustou-se o modelo de distribuição de probabilidades de Gumbel para o cálculo anual das precipitações máximas diárias por meio da seguinte sequência de equações (Gumbel, 1941; Pinto, 1995):

$$
\begin{aligned}
& \mathrm{Y}_{\mathrm{TR}}=-\ln \left[-\ln \left(1-\frac{1}{\mathrm{TR}}\right)\right] \\
& \mathrm{K}_{\mathrm{TR}}=-0,45+0,78 \cdot \mathrm{Y}_{\mathrm{TR}} \\
& \mathrm{X}_{\mathrm{TR}}=\overline{\mathrm{x}}+\mathrm{K}_{\mathrm{TR}} \cdot \mathrm{S}
\end{aligned}
$$

em que $Y_{T R}$ é a variável reduzida de Gumbel; TR é o período de retorno (anos); $\mathrm{X}_{\mathrm{TR}}$ é a precipitação máxima diária para determinado $\mathrm{TR}(\mathrm{mm}) ; \mathrm{K}_{\mathrm{TR}}$ é o fator de frequên- 
cia (adimensional); $\overline{\mathrm{x}}$ e $\mathrm{S}$ são, respectivamente, a média das precipitações máximas diárias ( $\mathrm{mm}$ ) e o desvio-padrão das precipitações máximas diárias $(\mathrm{mm})$. O período de retorno também pode ser denominado de tempo de recorrência ou tempo de retorno e corresponde ao tempo médio decorrido entre as ocorrências de um evento, no caso a precipitação, que exceda ou iguale certa magnitude (Tucci, 1993).

\subsection{Análise dos dados nos postos pluviométricos da Agência Nacional de Águas}

Para a avaliação da magnitude das alturas pluviométricas observadas na Região Serrana, procedeu-se ao levantamento das séries históricas de chuvas diárias disponibilizadas no banco de dados HidroWeb (ANA, 2016).

Cabe destacar a importância da utilização de séries históricas longas, já que quanto maior a série, mais confiável será o resultado da análise estatística, capturando as variações nas tendências de chuvas ao longo do tempo.

Nas Tabelas 1 a 3 foram listados os postos selecionados, bem como o tamanho da série histórica de cada um. Como pode ser observado, a maioria dos postos pluviométricos cadastrados possui falhas significativas.

No estudo de eventos de chuva, as precipitações devem ser extraídas de séries históricas locais, sendo o ideal o uso de no mínimo 30 anos de dados (Tucci, 1993). No entanto, no caso da Região Serrana optou-se por utilizar todos os postos com mais de 10 anos de dados disponíveis no HidroWeb, devido à escassez de informações.

Estimativa do tempo de retorno das chuvas de $24 \mathrm{~h}$ nos postos pluviométricos da Agência Nacional de Águas

Após a seleção dos postos pluviométricos, foram levantadas as séries históricas de modo a coletar os dados das chuvas máximas diárias de cada ano, sem considerar os anos que apresentassem falhas de observação.

Em seguida, para obtenção dos valores de precipitação máxima com duração de 24 h (P24), os dados máximos diários anuais (Pdia) foram desagregados a partir de fator de proporcionalidade, também denominado de desagregação da chuva diária.

A desagregação se inicia a partir da relação entre as alturas pluviométricas das chuvas P24 e Pdia, que segundo Cardoso et al. (1998), é uma relação quase constante, independentemente do período de retorno. De acordo com Occhipinti e Santos (1966), utilizando séries de dados de 1928 a 1965 de São Paulo, a relação varia entre 1,13 a 1,15, com média de 1,14. Já Genovez e Pegoraro (1998) obtiveram o valor médio de 1,13, igual ao definido pelo U.S. Weather Bureau (Tucci, 1993).

Análise dos Dados Pluviométricos do Sistema de Alerta de Cheias do Instituto Estadual do Ambiente

Tabela 1 - Postos pluviométricos existentes em Teresópolis na base da ANA.

\begin{tabular}{|c|c|c|c|c|c|c|c|}
\hline \multirow[t]{2}{*}{ Código } & \multirow[t]{2}{*}{ Nome do Posto } & \multicolumn{2}{|c|}{ Coordenadas } & \multirow[t]{2}{*}{ Altitude (m) } & \multicolumn{3}{|c|}{ Série histórica* } \\
\hline & & Latitude & Longitude & & Início & Fim & Tamanho (anos) \\
\hline 2242026 & Bom Sucesso & $-22,2714$ & $-42,7947$ & 870 & $01 / 11 / 1965$ & $01 / 12 / 2005$ & 31 \\
\hline 2242027 & Faz. Sobradinho & $-22,2011$ & $-42,9000$ & 650 & $01 / 05 / 1936$ & $01 / 12 / 2005$ & 60 \\
\hline 2242054 & Soberbo & $-22,4500$ & $-42,9833$ & 1004 & $01 / 01 / 1936$ & $01 / 05 / 1956$ & 10 \\
\hline 2242100 & Fazenda Coqueiro & $-22,4283$ & $-42,8008$ & 140 & $01 / 08 / 1978$ & $01 / 12 / 1993$ & 15 \\
\hline
\end{tabular}

*Anos com falhas observadas: Bom Sucesso - 1978 a 1981, 1996 a 2000; Fazenda Sobradinho - 1978 a 1981, 1996 a 2000; Soberbo - 1937,1941 a 1949.

Tabela 2 - Postos pluviométricos existentes em Nova Friburgo na base da ANA.

\begin{tabular}{|c|c|c|c|c|c|c|c|}
\hline \multirow[t]{2}{*}{ Código } & \multirow[t]{2}{*}{ Nome do Posto } & \multicolumn{2}{|c|}{ Coordenadas } & \multirow[t]{2}{*}{ Altitude (m) } & \multicolumn{3}{|c|}{ Série histórica* } \\
\hline & & Latitude & Longitude & & Início & Fim & Tamanho (anos) \\
\hline 2242003 & Piller & $-22,4047$ & $-42,3392$ & 670 & $01 / 08 / 1950$ & $01 / 12 / 2005$ & 53 \\
\hline 2242004 & Galdinópolis & $-22,3636$ & $-42,3808$ & 740 & $01 / 08 / 1950$ & $01 / 07 / 2006$ & 54 \\
\hline 2242005 & Faz. São João & $-22,3903$ & $-42,4947$ & 960 & $01 / 05 / 1967$ & $01 / 12 / 2005$ & 34 \\
\hline 2242020 & Vargem Grande & $-22,2761$ & $-42,5039$ & 680 & $01 / 12 / 1965$ & $01 / 12 / 2005$ & 36 \\
\hline 2242022 & Fazenda Mendes & $-22,2858$ & $-42,6600$ & 1010 & 01/06/1949 & $01 / 12 / 2005$ & 52 \\
\hline 2242023 & Conselheiro Paulino & $-22,2167$ & $-42,5167$ & 830 & $01 / 11 / 1938$ & $01 / 04 / 1977$ & 36 \\
\hline 2242024 & Teodoro de Oliveira & $-22,3772$ & $-42,5517$ & 1105 & 01/01/1982 & $01 / 12 / 2005$ & 18 \\
\hline 2242025 & Cascatinha do Conego & $-22,3425$ & $-42,5572$ & 1210 & $01 / 01 / 1982$ & $01 / 12 / 2005$ & 16 \\
\hline 2242032 & Nova Friburgo & $-22,2833$ & $-42,5333$ & 830 & 01/01/1938 & $01 / 05 / 1950$ & 11 \\
\hline
\end{tabular}

*Anos com falhas observadas: Piller - 1987 e 1988; Galdinópolis - 1953 e 1959; Fazenda São João - 1967, 1984, 2003, 2005; Vargem Grande - 1965, 1976, 1984, 2005; Fazenda Mendes - 1949, 1984, 1987, 2005; Conselheiro Paulino - 1976, 1980, 1981; Teodoro de Oliveira - 1996 a 2000; Cascatinha Conego - 1967, 1984, 1985, 1993, 1994, 2005, 2011, 2015; Nova Friburgo - 1950. 
Tabela 3 - Postos pluviométricos existentes em Petrópolis na base da ANA.

\begin{tabular}{|c|c|c|c|c|c|c|c|}
\hline \multirow[t]{2}{*}{ Código } & \multirow[t]{2}{*}{ Nome do Posto } & \multicolumn{2}{|c|}{ Coordenadas } & \multirow[t]{2}{*}{ Altitude (m) } & \multicolumn{3}{|c|}{ Série histórica* } \\
\hline & & Latitude & Longitude & & Início & Fim & Tamanho (anos) \\
\hline 2243009 & Petrópolis & $-22,5117$ & $-43,17083$ & 890 & $01 / 05 / 1938$ & $01 / 08 / 2005$ & 35 \\
\hline 2243010 & Itamarati (SE) & $-22,4853$ & $-43,1492$ & 1085 & 01/07/1938 & $01 / 12 / 2005$ & 56 \\
\hline 2243011 & Rio da Cidade & $-22,4381$ & $-43,1703$ & 704 & $01 / 07 / 1938$ & $01 / 12 / 2005$ & 58 \\
\hline 2243012 & Pedro do Rio & $-22,3325$ & $-43,1361$ & 645 & $01 / 11 / 1938$ & $01 / 12 / 2005$ & 52 \\
\hline 2243014 & Fagundes & $-22,2997$ & $-43,1781$ & 460 & $01 / 07 / 1954$ & $01 / 12 / 2005$ & 25 \\
\hline 2243016 & $\begin{array}{l}\text { Moreli (Parada } \\
\text { Moreli) }\end{array}$ & $-22,1931$ & $-43,0083$ & 600 & $01 / 01 / 1955$ & $01 / 12 / 2005$ & 32 \\
\hline
\end{tabular}

*Anos com falhas observadas: Petrópolis - 1938, 1951 a 1955, 1959, 1963, 1964, 1966 a 1971, 1973 a 1975, 1977 a 1980, 1984, 1985, 1992, 1996 a 2000 , 2003, 2005; Itamarati (SE) - 1938, 1978 a 1981, 1983, 1984, 1996 a 1999, 2000, 2005; Rio da Cidade - 1938, 1978 a 1981, 1996 a 2000 ; Pedro do Rio 1938, 1961, 1978 a 1981, 1984, 1987, 1991, 1992, 1995 a 2000; Fagundes - 1954, 1957, 1961 a 1967, 1971, 1977 a 1985, 1990, 1993, 1994,1996 a 2000; Moreli (Parada Moreli) - 1964, 1978, 1979 a 1981, 1984, 1986 a 1990, 1993 a 2000; 2005.

Foram também levantadas as informações existentes na base de dados do Sistema de Alerta de Cheias do Instituto Estadual do Ambiente (Inea) para identificação das chuvas P24 dos eventos de 2011 e 2013. As datas de início de operação, bem como as coordenadas de localização, dos postos pluviométricos dos municípios de Teresópolis, Nova Friburgo e Petrópolis são mostradas nas Tabelas 4 a 6.

Da observação das Tabelas, verifica-se que os postos pluviométricos do Sistema de Alerta do Inea, nos municí-

Tabela 4 - Localização dos postos pluviométricos do Sistema de Alerta do Inea em Teresópolis.

\begin{tabular}{lccc}
\hline \multirow{2}{*}{ Nome da estação } & $\begin{array}{c}\text { Data de } \\
\text { instalação }\end{array}$ & \multicolumn{2}{c}{ Coordenadas } \\
\cline { 3 - 4 } & & Latitude & Longitude \\
\hline Caleme & $16 / 11 / 2011$ & $-22,401767$ & $-43,012014$ \\
Comari & $07 / 10 / 2011$ & $-22,445917$ & $-42,975806$ \\
Posse - São Sebastião & $12 / 11 / 2011$ & $-22,373103$ & $-43,000967$ \\
Quebra Frascos & $27 / 01 / 2012$ & $-22,417056$ & $-43,007664$ \\
Unifeso & $07 / 11 / 2011$ & $-22,419394$ & $-42,967000$ \\
\hline
\end{tabular}

Tabela 5 - Localização dos postos pluviométricos do Sistema de Alerta do Inea em Nova Friburgo.

\begin{tabular}{lccc}
\hline \multirow{2}{*}{ Nome da estação } & \multirow{2}{*}{$\begin{array}{c}\text { Data de } \\
\text { instalação }\end{array}$} & \multicolumn{2}{c}{ Coordenadas } \\
\cline { 3 - 4 } & & Latitude & Longitude \\
\hline Conselheiro Paulino & $01 / 11 / 2008$ & $-22,228464$ & $-42,520136$ \\
Olaria & $01 / 11 / 2008$ & $-22,308842$ & $-42,542211$ \\
Pico Caledônia & $01 / 11 / 2008$ & $-22,359197$ & $-42,567367$ \\
Suspiro & $01 / 11 / 2008$ & $-22,279564$ & $-42,534822$ \\
Venda das Pedras & $01 / 11 / 2012$ & $-22,278464$ & $-42,581531$ \\
Ypu & $01 / 11 / 2008$ & $-22,295858$ & $-42,526503$ \\
Galdinópolis & $29 / 01 / 2011$ & $-22,369083$ & $-42,379444$ \\
Macaé de Cima & $25 / 04 / 2012$ & $-22,372389$ & $-42,462472$ \\
Piller & $25 / 04 / 2012$ & $-22,409083$ & $-42,336056$ \\
\hline
\end{tabular}

pios de Teresópolis e Petrópolis, foram instalados no segundo semestre de 2011, após o desastre ocorrido em janeiro. Já em Nova Friburgo, o início do monitoramento data de 2008, entretanto, no evento de janeiro de 2011, os postos Conselheiro Paulino, Venda das Pedras, Galdinópolis, Macaé de Cima e Piller apresentaram ausência de dados coletados.

Por último, cabe destacar que serão comparados apenas os postos pluviométricos da ANA e do Inea que possuírem a mesma localização ou muito próxima. No caso específico da cidade de Petrópolis, tendo em vista o relevo da região bem como o total precipitado observado no posto

Tabela 6 - Localização dos postos pluviométricos do Sistema de Alerta do Inea em Petrópolis.

\begin{tabular}{lccc}
\hline \multirow{2}{*}{ Nome da estação } & \multirow{2}{*}{$\begin{array}{c}\text { Data de } \\
\text { instalação }\end{array}$} & \multicolumn{2}{c}{ Coordenadas } \\
\cline { 3 - 4 } & & Latitude & Longitude \\
\hline Alto da Serra & $06 / 10 / 2011$ & $-22,513833$ & $-43,172917$ \\
Araras & $01 / 10 / 2011$ & $-22,383889$ & $-43,075833$ \\
Barão do Rio Branco & $17 / 10 / 2011$ & $-22,488167$ & $-43,177667$ \\
Bingen & $01 / 10 / 2011$ & $-22,509278$ & $-43,195694$ \\
Bonfim & $01 / 10 / 2011$ & $-22,461333$ & $-43,095167$ \\
Capim Roxo & $01 / 10 / 2011$ & $-22,351611$ & $-43,191556$ \\
Centro & $01 / 10 / 2011$ & $-22,512472$ & $-43,180278$ \\
Coronel Veiga & $25 / 10 / 2011$ & $-22.526266^{\circ}$ & $-43.193837^{\circ}$ \\
Cuiabá & $04 / 05 / 2011$ & $-22.369794^{\circ}$ & $-43.068569^{\circ}$ \\
Independência & $31 / 10 / 2011$ & $-22,548028$ & $-43,208917$ \\
Itaipava & $04 / 11 / 2011$ & $-22,405806$ & $-43,102944$ \\
Itamarati & $13 / 10 / 2011$ & $-22,484972$ & $-43,150250$ \\
LNCC & $10 / 06 / 2011$ & $-22,530278$ & $-43,217222$ \\
Morin & $14 / 10 / 2011$ & $-22,526611$ & $-43,160889$ \\
Pedro do Rio & $01 / 09 / 2011$ & $-22,333444$ & $-43,133000$ \\
Posse & $01 / 09 / 2011$ & $-22,258111$ & $-43,076361$ \\
Quitandinha & $26 / 10 / 2011$ & $-22,529444$ & $-43,223500$ \\
Samambaia & $01 / 09 / 2011$ & $-22,460111$ & $-43,141028$ \\
\hline & & &
\end{tabular}


Quitandinha do Inea, esta proximidade foi representada por um raio de $3 \mathrm{~km}$ a partir do posto Petrópolis da ANA, como ilustrado na Fig. 1.

\section{Resultados e Discussões}

Os postos pluviométricos da ANA que possuem a mesma localização ou muito próxima aos postos do Sistema de Alerta de Cheias do Inea estão apresentados na Tabela 7.

Para cada um dos postos da ANA, foram levantados os maiores valores diários de precipitação em cada ano,

Tabela 7 - Postos pluviométricos da ANA próximos aos do Sistema de Alerta de Cheias do Inea.

\begin{tabular}{lcc}
\hline Inea & ANA & Município \\
\hline Comari & Soberbo & Teresópolis \\
Galdinópolis & Galdinópolis & Nova Friburgo \\
Piller & Piller & Nova Friburgo \\
Itamarati & Itamarati-SE & Petrópolis \\
Pedro do Rio & Pedro do Rio & Petrópolis \\
$\begin{array}{l}\text { Alto da Serra; Bigen; Centro; } \\
\text { Coronel Veiga; Morin }\end{array}$ & Petrópolis & Petrópolis \\
\hline
\end{tabular}

adotada a conversão de Pdia em P24 a partir do fator de desagregação igual a 1,13 e ajustada a distribuição de Gumbel para estimativa das precipitações com tempos de recorrência de 5, 10, 20, 5, 100 e 1000 anos. A Tabela 8 apresenta os resultados encontrados.

Da análise da Tabela 8, observa-se que os postos Soberbo, Piller e Petrópolis apresentam valores semelhantes de precipitações, podendo ser considerados como representativos para estudos de análise de chuvas intensas. Um exemplo disso, é a sua comparação com os maiores eventos de $24 \mathrm{~h}$, anteriores ao de janeiro de 2011, citados por Lauriano et al. (2011), a saber: Teresópolis em 1961 com 161,6 mm, Nova Friburgo em 1964 com 113 mm e Petrópolis com $154,4 \mathrm{~mm}$ em 1947. No caso de Petrópolis, o evento possui a mesma ordem de grandeza da P24 associada a uma recorrência de 5 anos. Já para Nova Friburgo e Teresópolis, os eventos estão associados a uma recorrência abaixo de 5 anos.

Para os eventos dos dias 11 e 12 de janeiro de 2011 e 17 e 18 de março de 2013, as chuvas diárias nos postos do Sistema de Alerta de Cheias do Inea foram estimadas pela soma acumulada com início às 0:00 he fim às 24:00 h. Já as chuvas máximas de $24 \mathrm{~h}$ foram calculadas através da soma móvel dos dados registrados. As Tabelas 9 e 10 consolidam os resultados encontrados.

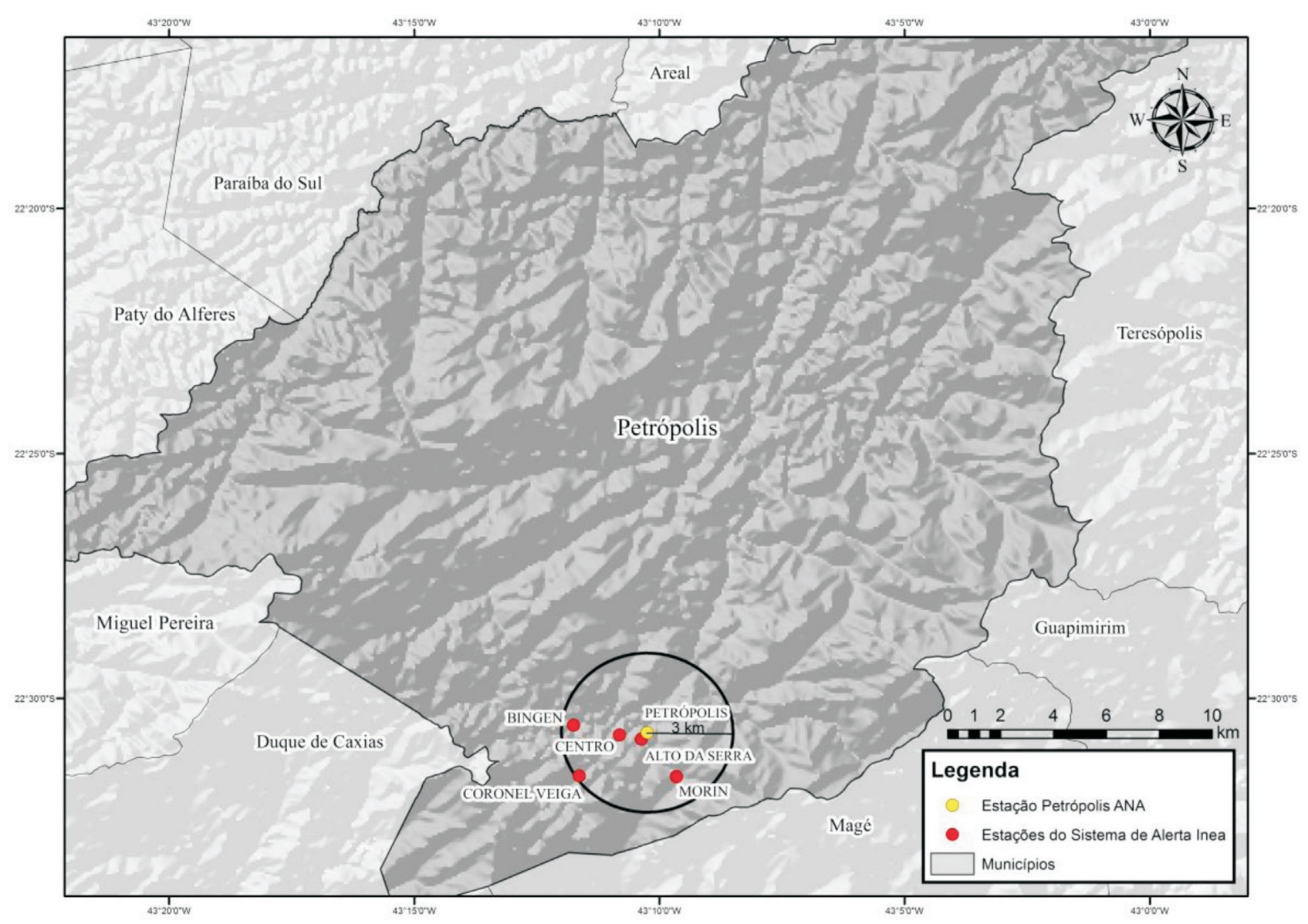

Figura 1 - Postos pluviométricos do Inea próximos ao posto Petrópolis da ANA. 
Tabela 8 - Recorrência das chuvas máximas de 24 h (P24), em mm.

\begin{tabular}{|c|c|c|c|c|c|c|c|}
\hline \multirow[t]{2}{*}{ Estação ANA } & \multirow[t]{2}{*}{ Município } & \multicolumn{6}{|c|}{ Tempo de recorrência (anos) } \\
\hline & & 5 & 10 & 20 & 50 & 100 & 1000 \\
\hline Soberbo & Teresópolis & 169,8 & 193,4 & 216,0 & 245,3 & 267,3 & 339,8 \\
\hline Galdinópolis & Nova Friburgo & 133,8 & 150,0 & 165,6 & 185,8 & 200,9 & 250,9 \\
\hline Piller & Nova Friburgo & 167,3 & 190,1 & 212,0 & 240,2 & 261,5 & 331,5 \\
\hline Petrópolis & Petrópolis & 157,2 & 183,5 & 208,7 & 241,4 & 265,9 & 346,9 \\
\hline Itamarati-SE & Petrópolis & 124,4 & 143,5 & 161,8 & 185,6 & 203,4 & 262,1 \\
\hline Pedro do Rio & Petrópolis & 99,4 & 112,4 & 124,9 & 141,1 & 153,3 & 193,3 \\
\hline
\end{tabular}

Tabela 9 - Pdia e P24 em Nova Friburgo nos dias 11 e 12 de janeiro de 2011.

\begin{tabular}{lcccc}
\hline Estação Inea & Pdia (Dia 11) $(\mathrm{mm})$ & Pdia $($ Dia 12) $(\mathrm{mm})$ & P24 (mm) & Data e hora de início da P24 \\
\hline Olaria & 225,20 & 44,40 & 245,20 & $11 / 01$ às 01:15 \\
Pico Caledônia & 165,00 & 13,00 & 173,40 & $11 / 01$ às 01:15 \\
Suspiro & 161,20 & 47,60 & 191,80 & $11 / 01$ às 02:45 \\
Ypu & 240,4 & 50,0 & 273,80 & $11 / 01$ às 02:00 \\
\hline
\end{tabular}

Tabela 10 - Pdia e P24 em Teresópolis, Nova Friburgo e Petrópolis nos dias 17 e 18 de março de 2013.

\begin{tabular}{|c|c|c|c|c|c|}
\hline Estação Inea & Município & Pdia (dia 17) (mm) & Pdia (dia 18) (mm) & $\mathrm{P} 24(\mathrm{~mm})$ & Data e hora de início da P24 \\
\hline Comari & Teresópolis & 126,60 & 156,80 & 271,40 & $17 / 03$ às $17: 45$ \\
\hline Galdinópolis & Nova Friburgo & 68,80 & 66,20 & 124,60 & $17 / 03$ às $15: 00$ \\
\hline Piller & Nova Friburgo & 49,80 & 211,80 & 220,00 & 17/03 às 20:30 \\
\hline Itamarati & Petrópolis & 112,25 & 14,50 & 123,75 & 17/03 às 09:15 \\
\hline Pedro do Rio & Petrópolis & 0,00 & 0,50 & 0,50 & 17/03 às 08:00 \\
\hline Alto da Serra & Petrópolis & 226,75 & 124,50 & 318,75 & $17 / 03$ às $12: 30$ \\
\hline Bingen & Petrópolis & 101,84 & 101,59 & 143,01 & 17/03 às 16:30 \\
\hline Centro & Petrópolis & 20,75 & 134,25 & 136,50 & 17/03 às 23:00 \\
\hline Coronel Veiga & Petrópolis & 266,25 & 183,00 & 408,25 & $17 / 03$ às $12: 30$ \\
\hline Morin & Petrópolis & 260,25 & 212,00 & 407,25 & $17 / 03$ às $12: 45$ \\
\hline
\end{tabular}

Da observação da Tabela 9, o evento ocorrido em 2011 teve seu período mais crítico das 2:00 h do dia 11 às 2:00 h do dia 12 de janeiro, totalizando $273,80 \mathrm{~mm}$ no posto Ypu. De forma análoga, da Tabela 10, no que diz respeito às precipitações de 2013, o evento teve seu período mais crítico das $12: 30 \mathrm{~h}$ do dia 17 às $12: 30 \mathrm{~h}$ do dia 18 de março, no posto Coronel Veiga em Petrópolis, com um total de 408,25 mm.

Para estimar os tempos de retorno do evento crítico de 2011, tendo em vista que o posto Ypu do Inea não está próximo a nenhum posto selecionado da ANA, foi necessário adotar o posto Piller, uma vez que, apesar da distância considerável, ele possui série histórica extensa com altos índices pluviométricos, como mencionado. Assim, ao inserir o valor $273,80 \mathrm{~mm}$ na Tabela 11 , referente aos resultados dos tempos de retorno do posto Piller, observa-se que o evento registrado no posto Ypu está associado a um tempo de retorno de 151,1 anos.
Já para o evento crítico de 2013, de acordo com a Tabela 7, o posto Soberbo da ANA foi identificado como sendo o mais próximo do posto Comari do Inea. Na avaliação da maior chuva P24 registrada em 2013, Comari apresentou um valor de 271,40 mm. Em comparação com a

Tabela 11 - Recorrência da P24 do posto Ypu em 2011 nas recorrências do posto Piller.

\begin{tabular}{lc}
\hline $\mathrm{T}($ anos $)$ & Chuva $\left(\mathrm{X}_{\mathrm{T}}\right)$ \\
\hline 5 & 167,3 \\
10 & 190,1 \\
20 & 212,0 \\
50 & 240,2 \\
100 & 261,5 \\
$\mathbf{1 5 1 , 1}$ & $\mathbf{2 7 3 , 8}$ \\
1000 & 331,5 \\
\hline
\end{tabular}


recorrência das chuvas de Soberbo, resultou em tempo de retorno de 115,2 anos.

A maior chuva P24 registrada em Nova Friburgo em 2013 foi observada no posto Piller do Inea e totalizou $220,00 \mathrm{~mm}$. Em comparação com a recorrência das chuvas do posto Piller da ANA, resultou em um tempo de retorno de 26,5 anos.

No caso de Petrópolis, a maior chuva P24 registrada em 2013 no posto Coronel Veiga do Inea foi igual a 408,25 mm. Em comparação com a recorrência das chuvas do posto Petrópolis, da ANA, resultou em um tempo de recorrência acima de 5000 anos. Dada a grandiosidade do resultado, optou-se por ajustar fator de desagregação. Para tanto, foram calculadas as relações Pdia e P24, dos dias 17 e 18 de março de 2013, dos postos do Sistema de Alerta do Inea, como mostrado na Tabela 12.

Da Tabela 12, observa-se que, apesar da literatura indicar um fator 1,13 ou 1,14 , ele não representa adequadamente os dados observados nos postos de Petrópolis. $\mathrm{O}$ fator de desagregação obtido para o posto Coronel Veiga, que apresentou a maior chuva de $24 \mathrm{~h}$ no evento de 2013, foi igual a 1,53 .

Tal resultado, acima do preconizado pela literatura, também foi encontrado por Freitas et al. (2017) quando avaliaram as relações P24/Pdia em 17 estações pluviométricas do Sistema Alerta Rio, instaladas na cidade do Rio de Janeiro. De acordo com os autores, 12 estações apresentaram fatores variando entre 1,19 e 1,81 . Back et al. (2012), encontraram um valor médio de 1,24 nas estações pluviográficas localizadas no litoral de Santa Catarina. Ambos os trabalhos confirmaram a variabilidade espacial das chuvas, justificada pelas distintas formas de relevo existentes e, portanto, as relações deveriam ser específicas para cada localidade. Esta condição também foi apontada por Konrad II (1996), quando menciona que a complexidade dos padrões espaciais da chuva em terrenos montanhosos se dá pela circulação atmosférica interagindo com as diversas características topográficas e paisagísticas, incentivando a ocorrência de precipitação em determinados lugares, enquanto que inibe esses eventos em outras localidades.

Com base no exposto, foi novamente calculada a recorrência das chuvas P24 na estação Petrópolis da ANA e os resultados alcançados estão relacionados na Tabela 13.

Como a maior P24, observada no posto Coronel Veiga do Inea, totalizou $408,25 \mathrm{~mm}$, ao comparar este valor
Tabela 13 - Recorrência das chuvas máximas de 24 h (P24), em mm, no posto Petrópolis da ANA a partir do fator de desagregação de 1,53.

\begin{tabular}{lc}
\hline $\mathrm{T}$ (anos) & Chuva $\left(\mathrm{X}_{\mathrm{T}}\right)$ \\
\hline 5 & 212,81 \\
10 & 248,46 \\
20 & 282,64 \\
50 & 326,90 \\
100 & 360,05 \\
1000 & 469,63 \\
\hline
\end{tabular}

com a recorrência das chuvas do posto Petrópolis da ANA, resulta em um tempo de retorno de 135,2 anos.

Em resumo, para a cidade de Nova Friburgo foi observado que o tempo de recorrência do evento de 2011 foi de aproximadamente 150 anos. Já para as cidades de Petrópolis e Teresópolis, o evento de 2013 resultou em tempos de retorno iguais a 135 e 115 anos, respectivamente. Observa-se que, tanto em 2011 quanto em 2013, nas cidades estudadas ocorreram chuvas significativas com recorrências de mesma ordem de grandeza, entretanto os desastres associados a estes eventos resultaram em efeitos distintos.

Estes resultados são coerentes com as informações da Defesa Civil que indicaram que Nova Friburgo foi o município mais atingido em 2011, enquanto o foco dos escorregamentos, em 2013, foi o município de Petrópolis. Embora grave, o evento de 2013 não foi tão significativo quanto o de 2011, em número de mortes e escorregamentos.

\section{Conclusões}

De acordo com a análise do histórico de chuvas da Região Serrana, ambos os eventos de 2011 e 2013 foram consideravelmente acentuados. De maneira geral, a intensidade do evento ocorrido em Petrópolis em 2013 superou a intensidade das chuvas ocorridas em Nova Friburgo em 2011.

Os resultados das análises mostraram valores diferentes das relações $\mathrm{P} 24 \mathrm{~h} / \mathrm{Pdia}$ aos preconizados na literatura. Eles confirmam a variabilidade espacial das chuvas na Região Serrana, justificada pelas suas distintas formas de relevo.

Por último, recomenda-se que para a avaliação da criticidade de um desastre não se considere apenas o total precipitado, visto que fatores como relevo, umidade do solo

Tabela 12 - Relação P24 e Pdia em Petrópolis.

\begin{tabular}{|c|c|c|c|c|}
\hline Estação Inea & Pdia $17(\mathrm{~mm})$ & Pdia $18(\mathrm{~mm})$ & $\mathrm{P} 24(\mathrm{~mm})$ & Fator de desagregação \\
\hline Itamarati & 112,25 & 14,50 & 123,75 & 1,10 \\
\hline Pedro do Rio & 0,00 & 0,50 & 0,5 & 1,00 \\
\hline Coronel Veiga & 266,25 & 183,00 & 408,25 & 1,53 \\
\hline
\end{tabular}


e ocupação de áreas de risco, bem como ocorrência de deslizamentos anteriores, podem ser determinantes na análise.

\section{Referências}

ANA. Agência Nacional de Águas - Banco de Dados HidroWeb: Séries Históricas. Disponível em: http://hidroweb.ana.gov.br. Acesso em 22 junho de 2016.

BACK, A.J.; OLIVEIRA, J.L.R.; HENN, A. Relações entre precipitações intensas de diferentes durações para desagregação da chuva diária em Santa Catarina. Rev. Bras. Eng. Agríc. Ambient v. 16, n. 4, p. 391-398, 2012.

BANCO MUNDIAL. Avaliação de perdas e danos: inundações e deslizamentos na Região Serrana do Rio de Janeiro em Janeiro de 2011. Relatório Técnico. Brasília: 2012. Disponível em: http://mi.gov.br/pt/c/document_library/get file?uuid 74dde46c-544a-4bc4-a6e1-852d4c09be06\&groupId = 10157. Acesso em 20 abril 2016.

BUSCH, A.; AMORIM, S. A tragédia da região serrana do Rio de Janeiro em 2011: procurando respostas. Casoteca de Gestão Pública, ENAP, 2011. Disponível em: http://casoteca.enap.gov.br/index.php?option=com_content $\&$ view $=$ article\&id

50:a-tragedia-da-regiao-serrana\&catid $=8$ : gestao-de-crise . Acesso em 3 agosto 2016.

CARDOSO, C.O.; ULLMANN, M.N.; BERTOL, I. Análise de chuvas intensas a partir da desagregação das chuvas diárias de Lages e de Campos Novos (SC). Rev. Bras. Ciênc. Solo, v. 22, n. 1, p. 131-140, 1998.

CARMO, R.L.; ANAZAWA, T.M. Mortalidade por desastres no Brasil: o que mostram os dados. Revista Brasileira de Ciência e Saúde Coletiva, Rio de Janeiro, v. 19, n. 9, p. 3669-3681, 2014.

CRED. Centre for Research on the Epidemiology of Disasters. Base de dados do EM-DAT - The International Disaster Database. Disponível em: http://www.emdat.be/. Acesso em 15 janeiro 2016.

DAMÉ, R.C.F.; TEIXEIRA, C.F.A.; MOREIRA, N.M.; SOUTO, M.V. Análise de frequência hidrológica dos dados de precipitação pluvial de algumas estações agroclimatológicas da região sul do Rio Grande do Sul. Cienc. Rural, v. 26, n. 3, p. 351-355, 1996.

DRM-RJ. Serviço Geológico do Estado do Rio de Janeiro. Correlação de Chuvas x Escorregamentos no Estado do Rio de Janeiro no mês de março de 2013. Relatório Técnico. Rio de Janeiro: 2013. Disponível em: http://www.drm.rj.gov.br/index.php. Acesso em 20 abril 2016.

ELTZ, F.L.; REICHERT, J.M.; CASSOL, E.A. Período de retorno de chuvas em Santa Maria, RS. R. bras. Ci. Solo, v. 16, p. 265-269, 1992.

FREITAS, F.S; HORA, M.A.G.M.; DUMAS, B.P. Análise das chuvas máximas diárias e de 24 horas na cidade do Rio de Janeiro. Revista SODEBRAS, Rio de Janeiro, v. 12, n. 135, p. 97-101, 2017.
GENOVEZ, A.M.; PEGORARO, R.C.F. Análise e avaliação de equações de chuvas intensas generalizadas. In: Congresso Latinoamericano de Hidráulica 18, 1998, Oaxaca. Anais... Oaxaca: IAHR, v. 1, 1998, p. 369-379.

GUMBEL, E.J. The Return Period of Flood Flows. Ann. Math. Statist., v. 12, n. 2, p. 163-160, 1941.

HARTMANN, M.; MOALA, F.A.; MENDONÇA, M.A. Estudo das precipitações máximas anuais em Presidente Prudente. Rev. Bras. Meteorol, v. 26, n. 4, p. 561$568,2011$.

HERSHFIELD, D.M. Rainfall Frequency Atlas of the United States for Durations from 30 Minutes to 24 Hours and Return Periods from 1 to 100 Years. Technical Paper No. 40, U.S. Weather Bureau, May 1961, 65 pp.

HUFF, F.A.; NEILL, J.C. Comparison of several methods for rainfall frequency analysis. J. Geophys. Res., v. 64 , n. 5, p. 541-547.

KONRAD II, C.E. Relationships between precipitation event types and topography in the southern blue ridge mountains of the Southeastern USA. Int. J. Climatol., v. 16, n. 1, p. 4962, 1996.

LAURIANO, C.; FREIRE, A.; LETA, T. Chuva espalha destruição na Região Serrana do Rio de Janeiro. O Globo, Rio de Janeiro, 12 de janeiro de 2011. Disponível em: http://g1.globo.com/rio-de-janeiro/noticia/2011/01/chuva-e spalha-destruicao-na-regiao-serrana-do-rio-de-janeiro.html Acesso em $1^{\circ}$ agosto 2016.

MAFRA, C.Q.T.; MAZZOLA, M. As razões dos desastres em território brasileiro. In: SANTOS, R.F. Vulnerabilidade Ambiental: Desastres naturais ou fenômenos induzidos? Brasília, DF: Ministério do Meio Ambiente, 2007. Disponível em: http://fld.com.br/uploads/documentos/pdf/Vulnerabilidade _Ambiental_Desastres_Naturais_ou_Fenomenos_Induzido s.pdf. Acesso em 27 julho 2017.

OCCHIPINTI, A.G.; SANTOS, P.M. Relações entre as precipitações máximas de "um dia" e de " 24 horas" na cidade de São Paulo. Instituto Astronômico e Geofísico, São Paulo, 26 p. 1966.

PFAFSTETTER, O. Chuvas Intensas no Brasil: relação entre precipitação, duração e frequência de chuvas em 98 postos com pluviógrafos. Rio de Janeiro: Departamento Nacional de Obras de Saneamento, 1957. 419 p.

PINTO, F.A. Chuvas intensas no Estado de Minas Gerais: análises e modelos. 1995. $87 \mathrm{f}$. Tese (Doutorado em Engenharia Agrícola) - Universidade Federal de Viçosa, Viçosa, 1995.

PINTO, F.R.L. Equações de intensidade-duração-frequência da precipitação para os estados do Rio de Janeiro e Espírito Santo: estimativa e espacialização. 1999. 70 p. Dissertação (Mestrado em Engenharia Agrícola) - Programa de Pós-Graduação em Engenharia Agrícola. Universidade Federal de Viçosa, Viçosa, 1999.

RIO DE JANEIRO (Estado). Secretaria do Ambiente. Assessoria de Comunicação do Inea. Inea desloca pessoal e máquinas para socorrer municípios atingidos pelas chuvas. Rio de Janeiro, 18 março 2013. Disponível em: http://www.rj.gov.br/web/imprensa/exibeconteudo?article-id $=1492505$. Acesso em 27 julho 2017 . 
RIO DE JANEIRO (Estado). Secretaria de Defesa Civil. Assessoria de Comunicação. Defesa Civil atualiza informações sobre as chuvas em Petrópolis. Rio de Janeiro, 22 março 2013. Disponível em: http://www.rj.gov.br/web/imprensa/exibeconteudo?article-id $=1500316$. Acesso em 27 julho 2017.

SILVA, D.D.; GOMES FILHO, R.R.; PRUSKI, F.F.; PEREIRA, S.B.; NOVAES, L.F. Chuvas intensas no Estado da Bahia. Rev. Bras. Eng. Agríc. Ambient., v. 6, n. 2, p. 362-367, 2002.

TUCCI, C.E.M. (Org.). Hidrologia Ciência e Aplicação. 1ª. ed. Porto Alegre: Editora da UFRGS e EDUSP, 1993. v. 1. $912 \mathrm{p}$.
THEBALDI, M.S. Análise estatística da precipitação máxima diária anual da cidade de Uberaba e vazão mínima diária anual do Rio Uberaba. Revista Agrogeoambiental, v. 4, n. 3, 2012.

WALDHERR, F.; TUPINAMBÁ, M.A. Dinâmica dos Depósitos Pretéritos na Deflagração de Corridas de Detritos em Eventos Catastróficos: A Bacia de Drenagem do Córrego do Príncipe, Teresópolis - RJ. In: VIII SLAGF Simpósio Latinoamericano de Geografia Física, IV SIAGF Simpósio Iberoamericano de Geografia Física. Anais. Santiago (Chile), 2014. p.1185-1192.

This is an Open Access article distributed under the terms of the Creative Commons Attribution Non-Commercial License which permits unrestricted non-commercial use, distribution, and reproduction in any medium provided the original work is properly cited 